\title{
Características demográficas e clínicas do grau de incapacidade física associadas ao diagnóstico e alta do tratamento da hanseníase
}

\author{
Demographic and clinical characteristics of the degree of physical disability associated \\ with the diagnosis and discharge from leprosy treatment
Características demográficas y clínicas del grado de discapacidad física asociada al diagnóstico y al tratamiento de la leprosa alta

\begin{abstract}
Laís Cristina Gonçalves Ribeiro ${ }^{1 *}$, Luana Oliveira da Rocha ${ }^{1}$, Natacha Bolorino ${ }^{1}$, Jaqueline Meira Uelse dos Santos ${ }^{1}$, Natalia Marciano de Araujo Ferreira1', Ricardo Alexandre Arcêncio², Antônio Carlos Vieira Ramos ${ }^{2}$, Franciely Midori Bueno de Freitas ${ }^{1}$, Carla Fernanda Tiroli ${ }^{1}$, Flávia Meneguetti Pieri ${ }^{1}$.
\end{abstract}

\section{RESUMO}

Objetivo: Verificar a associação entre as características demográficas e clínicas com o grau de incapacidade física no momento do diagnóstico e alta do tratamento de hanseníase. Métodos: Trata-se de um estudo transversal, analítico exploratório, de base populacional, utilizando dados secundários de casos de hanseníase ocorridos entre os anos de 2009 a 2017, extraídos do Sistema de Informação de Agravos de Notificação, conduzido em um município do norte do Paraná. Resultados: Constatou-se que $70,4 \%$ dos casos novos notificados com hanseníase foram identificados com incapacidade física no momento do diagnóstico, sendo o sexo masculino $(p=0,02)$ e $\circ$ tratamento padrão da poliquimioterapia $(p<0,01)$ estatisticamente significantes para o desenvolvimento do grau de incapacidade física (GIF) no momento da alta, considerando o GIF no diagnóstico. Conclusão: A evolução para incapacidades físicas na hanseníase reflete a qualidade dos serviços de saúde em relação a precocidade do diagnóstico e início do tratamento, sendo a Atenção Primária à Saúde a principal porta de entrada para este fim.

Palavras-chave: Hanseníase, Prevenção, Pessoa com incapacidade física, Saúde pública, Epidemiologia.

\begin{abstract}
Objective: To verify the association between demographics and the clinicals characteristics with the degree of physical incapacity at the time of diagnosis and the discharged from leprosy treatment. Methods: This is a cross-sectional population-based and analytical exploratory study, using secondary data from cases of leprosy in the period from 2009 to 2017. It was used the Notifiable Health Conditions Information System and it was coordinated in municipality of the Northern Paraná region. Results: It was verified that $70,4 \%$ of new cases reported with leprosy were identified with physical disability at the time of diagnosis, being the male gender $(p=0,02)$ and the official polychemotherapy to treat leprosy $(p<0,01)$ statistically significant results to developing the degree of physical disability at discharged, considering the degree of physical disability at diagnosis. Conclusion: The evolution to physical disabilities in leprosy reflect the quality of health services, considering the early diagnosis and the start of treatment, being the Primary Health Care the most important entrance for this purpose.
\end{abstract}

Key words: Leprosy, Prevention, Disable person, Public health, Epidemiology.

1 Universidade Estadual de Londrina (UEL), Londrina - PR. *E-mail: Icg.enf@hotmail.com

2 Universidade de São Paulo (USP), Ribeirão Preto - SP. 


\section{RESUMEN}

Objetivo: Verificar la asociación entre características demográficas y clínicas con el grado de discapacidad física en el momento del diagnóstico y del alta del tratamiento de lepra. Métodos: Se trata de un estudio transversal, analítico exploratorio, de base poblacional, que utiliza datos secundarios de casos de lepra ocurridos entre los años 2009 e 2017, sacados del Sistema de Información de Agravación de Notificación, realizado en un municipio del norte del Paraná. Resultados: Se constató que 70,4\% de los casos nuevos notificados con lepra fueron identificados con discapacidad física, siendo el sexo masculino $(p=0,02)$ y el tratamiento estándar de poliquimioterapia $(p<0,01)$ estadísticamente significativos para el desarrollo del grado de discapacidad física en el momento del alta, considerando el grado de discapacidad en el diagnóstico. Conclusión: La evolución para discapacidades físicas en la lepra refleja la calidad de los servicios de salud en relación con el diagnóstico precoz y el inicio del tratamiento, con la Atención Primaria de Salud siendo la principal vía de acceso para eso.

Palabras clave: Lepra, Prevención, Personas con discapacidad, Salud pública, Epidemiología.

\section{INTRODUÇÃO}

No último quadriênio, foi criado a Estratégia Global para Eliminação da Hanseníase 2016-2020, recomendando o fortalecimento da gestão do programa, enfrentamento da doença e eliminação com propósito de prevenir as incapacidades físicas, principalmente para indivíduos com idade igual ou menor a 15 anos e, por fim, acabar com o estigma, preconceito (OMS, 2016).

Em consonância com a recomendação internacional, o Ministério da Saúde (MS) estabeleceu a Estratégia Nacional para o Enfrentamento da Hanseníase (ENEH) 2019-2022, com as metas de redução progressiva entre os anos vigentes dessa estratégia para o grau de incapacidade física (GIF) II no momento do diagnóstico (BRASIL, 2019).

Nesse sentido, o MS classifica o GIF de acordo com a Classificação de Madri, que considera: GIF 0 não apresenta nenhuma manifestação clínica da hanseníase, GIF I apresenta alteração de sensibilidade em um segmento (olhos, mãos e pés) e GIF II apresenta alteração de sensibilidade em mais do que um segmento (BRASIL, 2020).

Para consolidar os pilares e metas propostas pelos esforços nacionais e internacionais, devido a especificidade da hanseníase, a Atenção Primária à Saúde (APS) configura-se como a principal porta de entrada para um efetivo e oportuno diagnóstico de hanseníase, contando com a equipe da Estratégia Saúde da Família (ESF) (SOUZA MM, et al., 2016).

Em vista disso, o momento do diagnóstico pode estar intimamente ligado com a evolução da doença, que tem alto poder incapacitante. Ressalta-se que além das transformações físicas, a hanseníase acarreta traumas psicológicos, sendo responsável pelo estigma e discriminação que estão associados à doença (PESCADOR MA, et al., 2018). Desta forma, torna-se fundamental o mapeamento dos casos de hanseníase com base no GIF identificado no momento do diagnóstico e alta, pela necessidade de produzir conhecimentos teóricos e operacionais, contribuindo assim, com a reflexão, discussão e concepção de estratégias de intervenção que subsidiem o adequado manejo da doença no contexto da rede de atenção à saúde, bem como o desenvolvimento de um processo cooperativo entre profissionais de saúde e usuários.

Diante do exposto, o objetivo desse estudo foi verificar a associação entre as características demográficas e clínicas com o grau de incapacidade física no momento do diagnóstico e alta do tratamento de hanseníase.

\section{MÉTODOS}

Trata-se de um estudo transversal, analítico exploratório, conduzido em um município do norte do Paraná, segunda cidade mais populosa do estado, com uma população estimada de 575.377 habitantes em 2020 (IBGE, 2020). 
O fluxo de atendimento aos casos de hanseníase é composto pela porta de entrada APS, constituída por 53 Unidades Básicas de Saúde (UBS), distribuídas em área urbana e rural; Policlínica, atuante no acompanhamento dos casos confirmados assistidos; Consórcio Intermunicipal de Saúde do Médio Paranapanema (CISMEPAR) que atua como referência para casos de maior complexidade e os acometidos com idade $\leq 15$ anos do município e/ou região (LONDRINA, 2020).

Para o desenvolvimento da pesquisa, considerou-se como população os 467 casos notificados/confirmados com diagnóstico de hanseníase, registrados no Sistema de Informações de Agravos de Notificação (SINAN), disponibilizados pela Secretaria de Vigilância Epidemiológica (SVE) do município.

Foram considerados os seguintes critérios de inclusão: casos confirmados de hanseníase notificados e acompanhados no município do estudo no período de janeiro de 2009 a dezembro de 2017 e como critérios de perdas as respostas faltantes e/ou incompletas no preenchimento das fichas de notificação.

Os dados foram coletados no período de agosto a outubro de $2018 \mathrm{com}$ apoio de um formulário específico elaborado para o presente estudo, contendo os seguintes eixos: Informações demográficas (sexo, faixa etária, raça/cor, escolaridade); Informações clínicas (classificação operacional, baciloscopia, número de lesões e número de nervos afetados, modo de entrada) e dados sobre o acompanhamento clínico, terapêutico e desfecho (esquema terapêutico, GIF no momento do diagnóstico e na alta).

Com relação às variáveis, foram realizados os tratamentos e criado categorias. A idade foi categorizada: faixa etária ( $\leq 16 ; 17$ a 59, $\geq 60$ anos); escolaridade: até 9 anos (ensino fundamental), de 10 a 12 anos (ensino médio) e $\geq 13$ anos (ensino superior); número de lesões: até cinco, de 6 a 20 e mais de 21; número de nervos afetados: até 2; 3 a 5 e mais de 6; raça/cor: branco/não branco.

As variáveis número de lesões e nervos afetados e forma clínica não foram utilizadas como repetição explicativas na associação entre por serem variáveis de confusão, visto que estão diretamente relacionadas com a variável classificação operacional.

Para análise, os dados foram transportados de uma planilha do Excel e analisados no IBM Software Statistical Package for the Social Science (SPSS) para o Windows e versão $20.0^{\circledR}$. Utilizou-se técnicas de análise descritiva para a caracterização do perfil demográfico e clínico dos casos.

Para a variável GIF no momento do diagnóstico e na alta foram utilizadas como categoria de referência, de forma a modelar os fatores que aumentam (ou diminuem) a probabilidade de ocorrência de GIF até o término do tratamento. Como variáveis de exposição, consideraram-se aquelas que apresentaram associação estatística significante para o teste Qui-quadrado (admitiu-se $p<0,05$ ). Estas foram inseridas conjuntamente no modelo.

Para verificar a associação entre o GIF no momento do diagnóstico e na alta dos casos de hanseníase construiu-se um modelo de regressão logística múltipla, com as variáveis preditoras faixa etária e forma clínica, analisados com relação aos fatores associados ao GIF na alta, com as variáveis: forma clínica, faixa etária, sexo e dose recebida da PQT.

Como critério para análise de regressão múltipla, adotou-se $p \leq 0,20$ para a inclusão da variável no modelo, segundo o teste da razão de Verossimilhança. Foi adotado o nível de significância de $p<0,05$ para todas as análises.

Para o desenvolvimento desta pesquisa, serão obedecidos os aspectos éticos e legais conforme Resolução 466/2012. Este estudo está vinculado a um projeto maior intitulado "A efetividade do sistema de saúde de Londrina no controle da hanseníase no período pré e pós-descentralização das ações para a atenção primária à saúde", com aprovação do Comitê de Ética em Pesquisa Envolvendo Seres Humanos da Universidade Estadual de Londrina (UEL), com CAAE 38642514.3.0000.5231 e Número do Parecer: 930.220.

\section{RESULTADOS}

Dos 467 casos novos notificados, 329 (70,4\%) foram identificados com GIF no momento do diagnóstico, GIF I 285 (61,0\%), GIF II 44 (9,4\%) e 19 (4,1\%) não foram avaliados ou estavam em branco. Ressalta-se que a variável escolaridade não foi classificada 155 (33,2\%) dos casos (Tabela 1). 
Tabela 1 - Características demográficas, clínico operacional dos casos notificados/confirmados de hanseníase.

\begin{tabular}{ccc}
\hline Variáveis & $\mathbf{N}$ & $\%$ \\
\hline Sexo $(\mathbf{n}=\mathbf{4 6 7})$ & & 56,3 \\
Masculino & 263 & 43,7 \\
\hline Feminino & 204 & \\
\hline Faixa Etária ( $\mathbf{n}=\mathbf{4 6 7 )}$ & & 3,2 \\
\hline até 16 anos & 15 & 67,7 \\
17 a 59 anos/ & 316 & 29,1 \\
60 ou mais & 136 & \\
\hline Raça/Cor (n=446) & & 71,3 \\
\hline Branco & 318 & 28,7 \\
\hline Não Branco & 128 & \\
\hline
\end{tabular}

\begin{tabular}{ccc}
\hline Escolaridade $(\mathbf{n = 3 1 2})$ & & 71,5 \\
\hline Até 9 anos de estudo & 223 & 21,1 \\
10 a 12 anos de estudo & 66 & 7,4 \\
Mais de 13 anos de estudo & 23 & 81,2 \\
\hline Classificação Operacional ( $\mathbf{n = 4 6 7 )}$ & 379 & 18,8 \\
\hline Multibacilar & 88 & 52,1 \\
Paucibacilar & & 36,4 \\
\hline Número de Lesões(n=459) & 239 & 11,5 \\
\hline Até 5 lesões & 167 & \\
De 6 a 20 lesões & 53 & 69,2 \\
Mais de 21 lesões & & 25,3 \\
\hline Número de Nervos Afetados $(\mathbf{n}=\mathbf{4 6 7 )}$ & 323 & 5,5 \\
\hline Até 02 nervos & 118 & \\
\hline De 03 a 05 nervos & 26 & \\
Mais de 06 nervos & &
\end{tabular}

Legenda: *Excluídos os casos ignorados/faltantes.

Fonte: Ribeiro LCG, et al., 2020.

Considerando as características específicas da doença, pode-se observar maior frequência de casos multibacilares (MB) $(379 ; 81,2 \%)$, com resultado de baciloscopia negativa $(52,9 \%)$ realizada no momento do diagnóstico. O modo de entrada mais frequente foi de casos novos com $69,8 \%$ (326), seguido de recidiva $14,6 \%(68)$, outros ingressos $7,7 \%$ (36), transferido de outro município $5,6 \%$ (26) e transferidos de outros estados $2,3 \%$ (11). 
Observando os casos de recidiva, notou-se uma crescente entre os anos de 2009 e 2015, correspondendo a (09/13,2\%) respectivamente. Em 2010, os casos de recidiva atingiram (02/2,9\%), em 2011 (05/7,4\%), seguido por $8,8 \%$ em 2012 e progredindo a (08/11,8\%) em 2013 . No ano de 2014 , os números voltaram a decrescer com (06/8,8\%), porém, os anos subsequentes revelaram um crescimento destes casos. No ano de 2015 , foram identificados $(09 / 13,2 \%)$ de recidivas de hanseníase, procedidas por $(13 / 19,1 \%)$ em 2016 e (16/14,07\%) em 2017.

Em relação ao percentual de detecção da hanseníase no período estudado, revelou-se que em 2017 houve uma diminuição de casos novos notificados. Apesar do declínio, o número ainda é considerado preocupante quando comparado aos anos anteriores (Figura 1).

Figura 1 - Distribuição do número de casos notificados/confirmados de hanseníase.

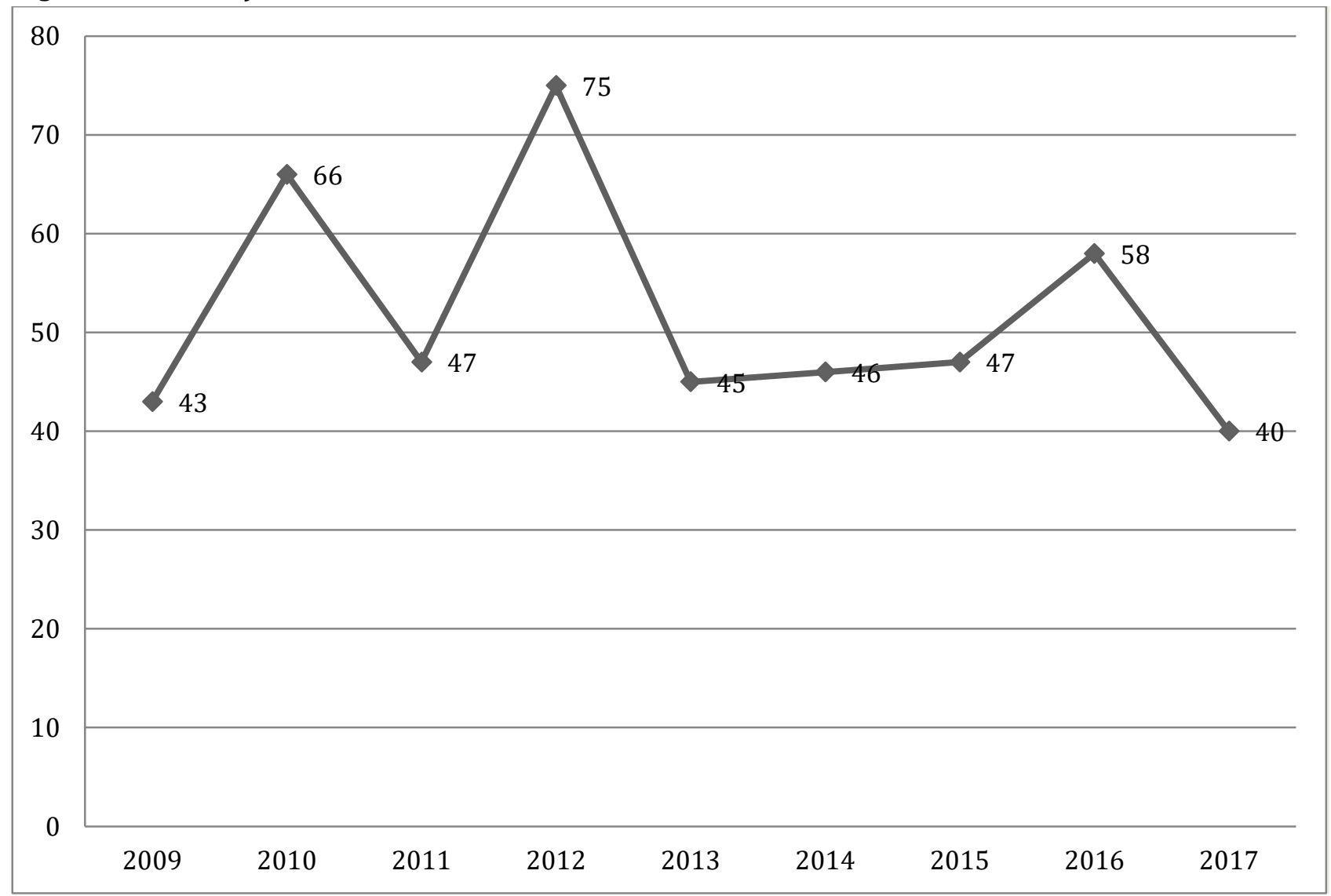

Fonte: Ribeiro LCG, et al., 2020.

Em relação ao GIF no momento do diagnóstico, foram considerados para efeito dos cálculos na caracterização da população os casos não avaliados e/ou em branco, totalizando (19/4,1\%) e os casos de GIF zero (120/25,7\%). Vale descrever que do total de casos de GIF zero, apenas um indivíduo não apresentava informação em algumas das variáveis, com isso o total passou a ser de 119 casos. O total da amostra dos casos com GIF no momento do diagnóstico foi de 70,4\% (329), sendo GIF I 61,0\% $(285)$ e GIF II 9,4\% (44) (Tabela 2).

Quanto ao GIF no momento da alta, foram ponderados os casos não avaliados e/ou em branco $(122 / 26,1 \%)$ e os casos de GIF zero (270/57,8\%). O total da amostra dos casos com GIF no momento da alta foi de $16,1 \%$ (75), sendo GIF I 5,0\% (23) e GIF II 11,1\% (52).

Desse modo, o GIF no diagnóstico e na alta foi apresentado como variável desfecho associadas às variáveis de exposição: forma clínica, faixa etária (apresentada em 06 categorias), sexo, tratamento anterior e doses recebidas da $P Q T$. 
Tabela 2 - Análise bivariada entre variáveis demográficas e clínicas, e a presença de incapacidade física no diagnóstico e na alta dos casos notificados/confirmados de hanseníase.

Incapacidade no Diagnóstico e na Alta

\begin{tabular}{lllllll}
\hline & Sim & Não & Valor-p & Sim & Não & Valor-p \\
\hline Forma Clínica* & & & & & & \\
\hline FI & $57,1 \%(20)$ & $42,9 \%(15)$ & $<0,01$ & $40,0 \%(14)$ & $40,0 \%(14)$ & \\
FT & $56,6 \%(30)$ & $39,6 \%(21)$ & & $47,1 \%(24)$ & $47,1 \%(24)$ & $<0,01$ \\
FD & $71,6 \%(159)$ & $25,2 \%(56)$ & & $53,0 \% 114)$ & $21,9 \%(47)$ & \\
FV & $76,4 \%(120)$ & $17,2 \%(27)$ & & $53,1 \%(78)$ & $14,3 \%(21)$ & \\
\hline
\end{tabular}

Faixa Etária**

\begin{tabular}{|c|c|c|c|c|c|c|}
\hline Até 16 anos & $20,0 \%(3)$ & $80,0 \%(12)$ & & $0,0 \%(0)$ & $86,7 \%(13)$ & 0,05 \\
\hline $17-27$ anos & $55,8 \%(24)$ & $44,2 \%(19)$ & & $14,0 \%(06)$ & $60,5 \%(26)$ & \\
\hline $28-38$ anos & $63,2 \%(43)$ & $35,3 \%(24)$ & & $22,1 \%(15)$ & $51,5 \%(35)$ & \\
\hline $39-49$ anos & $72,8 \%(75)$ & $23,3 \%(24)$ & $<0,01$ & $22,3 \%(23)$ & $61,2 \%(63)$ & \\
\hline $50-59$ anos & $74,5 \%(76)$ & $20,6 \%(21)$ & & $12,7 \%(13)$ & $57,8 \%(59)$ & \\
\hline$\geq 60$ anos & $79,4 \%(108)$ & $14,0 \%(19)$ & & $13,2 \%(18)$ & $54,4 \%(74)$ & \\
\hline \multicolumn{7}{|l|}{ Sexo } \\
\hline Masculino & $71,5 \%(188)$ & $23,2 \%(61)$ & 0,16 & $15,2 \%(40)$ & $56,3 \%(148)$ & 0,39 \\
\hline Feminino & $69,1 \%(141)$ & $28,4 \%(58)$ & & $17,2 \%(35)$ & $59,8 \%(122)$ & \\
\hline \multicolumn{7}{|c|}{ Tratamento Anterior } \\
\hline Não tratou & $56,7 \%(17)$ & $30,0 \%(9)$ & $<0,01$ & $13,3 \%(4)$ & $10,0 \%(3)$ & $<0,00$ \\
\hline Até 12 meses & $72,3 \%(204)$ & $25,5 \%(72)$ & & $14,5 \%(41)$ & $60,6 \%(171)$ & \\
\hline Até 24 meses & $73,9 \%(99)$ & $22,4 \%(30)$ & & $18,7 \%(25)$ & $65,7 \%(88)$ & \\
\hline Até 36 meses & $38,9 \%(7)$ & $38,9 \%(7)$ & & $27,8 \%(5)$ & $38,9 \%(7)$ & \\
\hline$\geq 37$ meses & $66,7 \%(2)$ & $33,3 \%(1)$ & & $0,0 \%(0)$ & $33,3 \%(1)$ & \\
\hline
\end{tabular}

\section{Esquema da PQT ${ }^{\star \star \star \star}$}

\begin{tabular}{lllllll}
\hline Padrão & $72,6 \%(220)$ & $25,1 \%(76)$ & 0,02 & $12,5 \%(38)$ & $82,5 \%(250)$ & \\
Substituto & $66,5 \%(109)$ & $26,2 \%(43)$ & & $22,6 \%(37)$ & $12,2 \%(20)$ & $<0,00$
\end{tabular}

Legenda: ${ }^{*} \mathrm{FI}=$ forma indeterminada, $\mathrm{FT}=$ forma tuberculóide, $\mathrm{FD}=$ forma dimorfa, $\mathrm{FV}=$ forma virchowiana; ${ }^{* *}$ Recategorização das faixas etárias; ${ }^{* \star *}$ Teste Qui-quadrado com correção de continuidade/Yates; ${ }^{* * * * P Q T ~-~}$ polimiquioterapia.

Fonte: Ribeiro LCG, et al., 2020.

Ao considerar os segmentos examinados, demonstrou-se que os pacientes com a Forma Tuberculóide (FT) apresentam 1,07 vezes mais chances de apresentarem incapacidades no diagnóstico quando comparado à pacientes com a Forma Clínica/Indeterminada (FI). Pacientes com a Forma Dimorfa (FD) apresentam 2,13 vezes mais chances de desenvolver incapacidades no diagnóstico do que pacientes com a Forma Indeterminada (FI). Ao analisar os pacientes com a FV, observa-se que há 3,33 vezes mais chances de desenvolver incapacidades no diagnóstico do que pacientes FI. Adicionalmente, o teste de Cox \&Snell demonstrou que a forma clínica pode explicar 3\% das incapacidades no diagnóstico (Tabela 3). 
Em relação à análise do GIF na alta, a FV apresentou 3,71 vezes mais chances de desenvolver incapacidades em relação a FI, procedida pela FD com 2,43 mais chances de desenvolver incapacidade ao término do tratamento $P Q T$.

Tabela 3 - Estimativas resultantes da aplicação do modelo de regressão logística múltipla para os casos com incapacidade física no momento do diagnóstico e na alta.

\begin{tabular}{lcl}
\hline \multicolumn{3}{c}{ Incapacidade física em hanseníase no diagnóstico } \\
\hline Forma Clínica** & OR $(\text { IC } 95 \%)^{\star}$ & p- Valor \\
\hline FT & $1,07(0,44-2,56)$ & 0,87 \\
FD & $2,13(1,02-4,44)$ & 0,04 \\
FV & $3,33(1,51-7,33)$ & $<0,01$ \\
FI & 1,00 & \\
\hline Faixa Etária & & \\
\hline Até 16 anos & 1,00 & 0,02 \\
$17-27$ anos & $5,05(1,24-20,51)$ & $<0,01$ \\
$28-38$ anos & $7,16(1,83-27,92)$ & $<0,01$ \\
$39-49$ anos & $12,50(3,25-48,02)$ & $<0,01$ \\
$50-59$ anos & $14,48(3,73-56,08)$ & $<0,01$ \\
$\geq 60$ anos & $22,74(5,86-88,22)$ & \\
\hline
\end{tabular}

\begin{tabular}{lll}
\hline \multicolumn{3}{c}{ Incapacidade física em hanseníase na alta } \\
\hline Forma Clínica** & OR $(\text { IC } 95 \%)^{*}$ & p-Valor \\
\hline FT & $1,00(0,39-2,54)$ & 1,00 \\
FD & $2,43(1,07-5,48)$ & 0,03 \\
FV & $3,71(1,53-8,98)$ & $<0,01$ \\
FI & 1,00 &
\end{tabular}

\section{Faixa Etária}

\begin{tabular}{lll}
\hline Até 16 & 1,00 & \\
$17-27$ anos & $6,23(1,19-32,75)$ & 0,03 \\
$28-38$ anos & $11,69(2,31-59,03)$ & $<0,01$ \\
$39-49$ anos & $12,69(2,63-61,33)$ & $<0,01$ \\
$50-59$ anos & $16,50(3,34-81,59)$ & $<0,01$ \\
$\geq 60$ anos & $18,33(3,77-89,32)$ & $<0,01$ \\
\hline Sexo & & 0,02 \\
\hline Masculino & $1,69(1,06-2,68)$ & \\
Feminino & 1,00 & $<0,01$ \\
\hline Esquema da PQT & \\
\hline Padrão & & \\
Substituto & $5,64(2,18-14,60)$ & \\
\hline
\end{tabular}

Legenda: ${ }^{*} \mathrm{Cl}$ : confidence interval, OR: odds ratio, $\mathrm{p}$-value $=<0,05 .{ }^{* *} \mathrm{Fl}=$ forma indeterminada, $\mathrm{FT}=$ forma tuberculóide, $\mathrm{FD}=$ forma dimorfa, $\mathrm{FV}=$ forma virchowiana. ${ }^{* \star *} \mathrm{PQT}$ - Poliquimioterapia

Fonte: Ribeiro LCG, et al., 2020. 


\section{DISCUSSÃO}

Esse estudo evidenciou que o sexo masculino $(p=0,02)$ e o tratamento padrão da $P Q T(p<0,01)$ foram estatisticamente significantes para o desenvolvimento do GIF no momento da alta, considerando o GIF no diagnóstico.

No que se refere à forma clínica, neste estudo, as formas FV e FD, classificadas operacionalmente como $\mathrm{MB}$, com ênfase para a FV, demonstraram ser potencialmente incapacitantes tanto no diagnóstico quanto na alta.

A FV está correlacionada à imunidade celular quase nula, o que predispõe a multiplicação do bacilo Mycobaterium leprae e a uma forma mais grave da hanseníase. Possui particularidades como hansenomas, de coloração acastanhada, principalmente em pavilhão auricular e/ou madarose nos supercílios e face leonina. Pode ainda apresentar outras complicações como o acometimento de testículo, suprarrenais, fígado, baço (BRASIL, 2017a).

Esta relação entre a carga bacilar e o GIF pode ser explicada pela maior gravidade da doença em relação à maior quantidade de bacilos encontrados. Dessa forma, altas taxas de indivíduos diagnosticados com a FV, sugerem presença de prevalência oculta da doença, ou seja, circulação de casos em estágio avançado de hanseníase, detectados tardiamente, que antes disso atuavam como difusores da endemia (BRASIL, 2020; FRANCISCO LLF, et al., 2019; OMS, 2016).

Esta afirmação intensifica a necessidade de detecção precoce da hanseníase, além da necessidade de os serviços de saúde priorizarem a avaliação ao diagnóstico, bem como valorizar os sinais e sintomas referidos pelos pacientes (BRASIL, 2017a; RIBEIRO GC e LANA FCF, 2015).

Ressalta-se que o para o diagnóstico de doenças crônicas como a hanseníase, a APS constitui-se como principal porta de entrada. Sob essa ótica de direção, destaca-se a importância de toda rede de atenção em saúde (RAS), em especial dos serviços da APS como recurso estratégico para alcançar a acessibilidade e descentralização da vigilância e do cuidado e consequentemente do enfrentamento da doença (BRASIL, 2017b).

Ainda, a APS e a Estratégia Saúde da Família (ESF) podem impactar positivamente, quando bem conduzidas, para a melhoria da situação epidemiológica, qualificação e consolidação do sistema, permitindo maior acessibilidade pelos usuários, em especial pelos considerados como vulneráveis.

À vista disso, com o intuito de consolidar a rede de atenção básica do Sistema Único de Saúde (SUS), o MS por meio da Portaria ํㅡ 149, de 3 de fevereiro de 2016, busca fortalecer o modelo de intervenção para o controle da hanseníase mediante aprovação das "Diretrizes para Vigilância, Atenção e Eliminação da Hanseníase como Problema de Saúde Pública", a fim de nortear profissionais de saúde e gestores garantindo atenção especializada no que concerne ao acolhimento saudável, diagnóstico precoce, tratamento em tempo oportuno e cura, minimizando consideravelmente a ocorrência de incapacidades físicas (BRASIL, 2016a).

Em se tratando ao nível de escolaridade, demonstrou-se que indivíduos com ausência ou menor nível educacional foram os mais acometidos pela hanseníase. Denota-se que níveis decrescentes de educação retratam condições de vida desfavoráveis, levando a condições sanitárias insatisfatórias para a manutenção de vida, a menor procura aos serviços de saúde e ao autocuidado, o que contribui para a manutenção da cadeia de transmissão da hanseníase, oportunizando o desenvolvimento das incapacidades físicas, principalmente o GIF II (NERY JS, et al., 2019; RIBEIRO GC e LANA FCF, 2015).

Estudo do tipo coorte conduzido por Nery JS, et al. (2019), em que foram analisadas 33 milhões de pessoas, das quais aproximadamente 24 mil foram diagnosticadas com hanseníase, possibilitou compreender que fatores como a baixa escolaridade favorecem a transmissibilidade do agravo. Essa afirmação é justificada pelo resultado encontrado, em que demonstram que indivíduos com baixo nível de escolaridade e/ou responsável familiar com baixa formação escolar, apresentaram incidência mais de duas vezes maior de contrair hanseníase do que comparados àqueles que concluíram os estudos além do Ensino Médio. 
Dessa forma, estimular o desenvolvimento social através da educação de qualidade pode ser uma estratégia para evitar com que esta parcela da população sofra os impactos negativos provenientes deste contexto social, visando o bem-estar e melhoria da qualidade de vida desses indivíduos (CELESTINO EP, et al., 2020).

Em relação ao sexo, o perfil masculino apresentou significância no GIF no momento da alta. Isso pode ser atribuído ao fator cultural de masculinidade atrelada nesta população, gerando resistência ao autocuidado e ao retardo na procura por atendimento clínico, favorecendo o diagnóstico tardio e consequente complicação da doença. Além disso, vale a pena mencionar que em se tratando da hanseníase, ainda há o estigma inerente à doença, fato que pode dificultar a procura por assistência em saúde (AGUIAR DX, et al., 2020; SOUZA EA, et al., 2018; GOIABEIRA YNLDA, et al., 2018; BASSO MEM e SILVA RLF, 2017).

No que concerne a faixa etária, é pertinente mencionar que foram expressivos os dados obtidos acerca da manifestação de incapacidades físicas em adultos em idade economicamente ativa tanto no momento do diagnóstico quanto na alta. Essa complicação acomete com mais frequência este grupo em virtude da alta exposição às atividades laborais, facilidade de contágio e à evolução lenta e silenciosa da doença, levando a um comprometimento físico que pode desenvolver limitação física e socioeconômica em razão dos afastamentos das atividades rentáveis (SANTANA MES, et al., 2020; BASSO MEM e SILVA RLF, 2017).

Entretanto, provocou inquietação os resultados obtidos em relação à presença de casos novos de hanseníase em indivíduos menores de 15 anos, assim como o acometimento por GIF I ou GIF II no diagnóstico nesta população. $O$ registro de casos nesta faixa etária é considerado o principal indicador de monitoramento do agravo pelo Programa Nacional de Controle da Hanseníase (PNCH). Esses dados sugestionam transmissão ativa e recente da doença, circulação do bacilo Mycobacterium leprae, falha diagnóstica, além de deficiência por parte dos serviços de saúde na atenção e no seguimento dos casos novos diagnosticados até o término da PQT e seus contatos sociais e intradomicilares (FREITAS BHBM, et al., 2017; OMS, 2016).

Além disso, a hanseníase pode causar diversos transtornos de ordem biopsicossocial nessa faixa etária. Por ser caracterizada pelas deformidades, lesões dermatológicas, alto poder incapacitante, pode prejudicar a construção de identidade desses indivíduos, e ainda, interferir negativamente na formação educacional, devido $o$ isolamento social em virtude do estigma, discriminação e o preconceito que são atribuídos à doença (SCHNEIDER PB e FREITAS BHBM, 2018).

No que tange a distribuição do número de casos notificados/confirmados de hanseníase, durante o período de estudo, verificou-se uma notável oscilação de detecção do agravo, atingindo em 2012, um aumento considerável de notificações e confirmações diagnósticas da doença, o que pode ser reflexo de uma subnotificação e subdetecção dos casos nos anos anteriores e posteriores

Uma justificativa plausível para a referida evolução do percentual de detecção da hanseníase, se deu em 2011, na semana da conscientização contra o agravo, reflexo de algumas ações desenvolvidas no município. Aconteceu na cidade de estudo vigilância ativa por meio de capacitação direcionada para profissionais de UBS e do Núcleo de Apoio à Saúde da Família (NASF) loco-regionais, promovida pela Secretaria Municipal de Saúde, $17^{\text {a }}$ Regional de Saúde, Consórcio Intermunicipal do Médio Paranapanema (Cismepar) e a Policlínica Municipal, com intuito de atualizar e qualificar profissionais da saúde sobre o diagnóstico precoce e o tratamento em tempo oportuno de pacientes com hanseníase (LONDRINA, 2011a).

Alunos do curso de enfermagem da Universidade Estadual de Londrina (UEL) igualmente contribuíram para a prevenção e controle da doença por meio de orientação à comunidade sobre técnicas de autocuidado e prevenção de deformidades e incapacidades físicas (LONDRINA, 2011b).

Considera-se essencial para prevenção de incapacidades o processo de educação em saúde. Demonstrar por meio de orientações de autocuidado que cada indivíduo é responsável pela manutenção do seu próprio bem-estar, provoca mudanças individuais, sustentando a construção de autonomia, favorecendo a mudança de hábitos sociais e de saúde (PINHEIRO MCG, et al., 2014). Nesse contexto, o início em tempo oportuno da PQT é igualmente relevante para impossibilitar a manifestação das incapacidades físicas. A terapia combinada fornece potente ação bactericida e bacteriostática ao eliminar e fragmentar o bacilo Mycobacterium leprae. Portanto, evita a evolução da doença e, consequentemente impede o desenvolvimento dessas complicações (BRASIL, 2016a) 
Sendo assim, em relação ao esquema PQT, este estudo demonstrou que pacientes que fizeram uso de esquema padrão para tratamento de hanseníase composto por dapsona, rifampicina e clofazimina, denotaram associação estatística significativa ao acometimento por GIF na alta comparados ao GIF no diagnóstico. Entretanto, não pode se desvincular que a forma clínica, adesão ao tratamento, trazer outros fatores que colaboram como GIF para não dar a entender que o PQT padrão leva ao GIF.

Desta maneira, é recomendado que todos os indivíduos acometidos por hanseníase sejam avaliados quanto ao GIF obrigatoriamente no momento do diagnóstico e alta do agravo, permitindo a realização de comparação entre os dois momentos, sendo relevante para definir a qualidade dos serviços em saúde prestados na APS (FERNANDES DOH, et al., 2019; BRASIL, 2017a).

De acordo com a Política Nacional de Atenção Básica (PNAB), é responsabilidade da atenção Básica (AB), tratar e acompanhar os indivíduos com hanseníase, bem como os contatos sociais e intradomicilares. Para uma maior efetividade das ações, as UBS com apoio da ESF contribuem com a vinculação e busca ativa dos usuários ao serviço por meio dos profissionais de saúde, em especial o ACS. Por consequência, é possível alcançar maior resolutividade aos cuidados, tratamento, educação em saúde, redução e controle dos casos, além de prevenção das incapacidades físicas, seja no momento do diagnóstico e/ou na alta da PQT (BRASIL, 2017b; BRASIL, 2016b).

A avaliação do GIF na alta foi considerada uma limitação do presente estudo devido ausência de registro ou avaliação do GIF nas fichas de notificação do SINAN entre alguns pacientes. Observou-se negligência da informação sobre esta avaliação no momento do diagnóstico de 4,9\% e de $26,1 \%$ na alta. Entretanto, em razão dos significantes resultados obtidos, acredita-se que esta pesquisa seja robusta e contribua com as estratégias da vigilância epidemiológica para alcançar uma tendência decrescente ao longo do tempo na proporção de pacientes que tem seu GIF agravado ou que evoluem com novas incapacidades durante o tratamento.

\section{CONCLUSÃO}

Este estudo permitiu verificar que o sexo masculino e a forma virchowiana tiveram associação com o desenvolvimento do GIF no momento da alta. Conhecer a hanseníase como doença de alto poder incapacitante em decorrência das complicações causadas por ela, as denominadas incapacidades físicas, bem como saber sobre seu perfil epidemiológico e clínico, compreendendo seu comportamento na comunidade por meio das relações e suas dependências entre as variáveis, se fazem relevantes para refletir sobre a ENEH em prol de uma assistência integral e de qualidade aos indivíduos acometidos por este agravo desde o diagnóstico até a alta por cura na APS.

\section{REFERÊNCIAS}

1. AGUIAR DX, et al. Perfil epidemiológico dos casos de hanseníase em Porto Nacional - Tocantins de 2007 a 2018. Revista Ciências em Saúde, 2020; 10(2): 43-50.

2. BASSO MEM, SILVA RLF. Perfil clínico-epidemiológico de pacientes acometidos pela hanseníase atendidos em uma unidade de referência. Revista da Sociedade Brasileira de Clínica Médica, 2017; 15(1): 27-32.

3. BRASIL MS. Secretaria de Vigilância em Saúde. Departamento de Doenças de Condições Crônicas e Infecções Sexualmente Transmissíveis. Hanseníase no Brasil: Caracterização das Incapacidades Físicas, 2020: 1-98.

4. BRASIL MS. Secretaria de Vigilância em Saúde. Estratégia Nacional para o Enfrentamento da Hanseníase-20192022, 2019: 1-16.

5. BRASIL MS. Secretaria de Vigilância em Saúde. Departamento de Vigilância das Doenças Transmissíveis. Guia prático sobre a hanseníase, 2017a: 1-70.

6. BRASIL MS. Portaria no 2.436. 2017b. In: Diário Oficial da União. Brasília. Disponível em: https://bvsms.saude.gov.br/bvs/saudelegis/gm/2017/prt2436_22_09_2017.html. Acesso em: 15 de out de 2020.

7. BRASIL MS. Portaria no 149. 2016a. In: Diário da União Brasília, Disponível em: http://bvsms.saude.gov.br/bvs/saudelegis/gm/2016/prt0149_04_02_2016.html. Acesso em: 21 de out de 2020.

8. BRASIL MS. Secretaria de Vigilância em Saúde. Diretrizes para vigilância, atenção e eliminação da Hanseníase como problema de saúde pública: manual técnico-operacional, 2016b: 1-60.

9. CELESTINO EP, et al. Educação e desenvolvimento social: reflexões que se intercruzam. Brazilian Journal of Development, 2020; 6(6): 35995-36007. 
10. CORRÊA GHLST, et al. Redes para o cuidado tecidas por idosa e família que vivenciam situação de adoecimento crônico. Revista Mineira de Enfermagem, 2014; 18(2): 356-364.

11. FERNANDES DOH, et al. Ocorrência das incapacidades físicas na hanseníase durante o tratamento medicamentoso. Rev. Bras. Pesq. Saúde, 2019; 21(3): 122-131.

12. FRANCISCO LLF, et al. Estimativa da prevalência oculta da hanseníase em município do interior do Estado de São Paulo. Arquivos de Ciências da Saúde, 2019; 26(9): 89-93.

13. FREITAS BHBM, et al. Tendência da hanseníase em menores de 15 anos em Mato Grosso (Brasil), $2001-2013$. Revista de Saúde Pública, 2017; 51(28): 1-10.

14. GOIABEIRA YNLDA, et al. Perfil Epidemiológico e clínico da hanseníase em capital hiperendêmica. Rev. enferm. UFPE on line, 2018; 12(6): 1507-1513.

15. IBGE. Instituto Brasileiro de Geografia e Estatística. Censo demográfico 2010: Informações completas. Londrina: IBGE, 2019. Disponível em: https://cidades.ibge.gov.br/brasil/pr/londrina/panorama. Acesso em: 31 de out de 2020.

16. NERY JS, et al. Socioeconomic determinants of leprosy new case detection in the 100 Million Brazilian Cohort: a population-based linkage study: a population-based linkage study. The Lancet Global Health, 2019; 7(9): $1226-1236$.

17. ORGANIZAÇÃO MUNDIAL DA SAÚDE. 2016. Estratégia mundial de eliminação da lepra 2016-2020: Acelerar a ação para um mundo sem lepra. Disponível em: https://apps.who.int/iris/bitstream/handle/10665/208824/9789290225201 pt.pdf;jsessionid=87BE71FC50CE01FDDFAFBC1D91459086? sequence=17. Acesso em: 25 set. 2020.

18. PÊGO AF, et al. Hanseníase: correlação entre o número de lesões hansênicas, nervos afetados e o diagnóstico precoce no estado de Minas Gerais. Revista Eletrônica Acervo Saúde, 2019; 12(9): 1-9.

19. PESCADOR MA, et al. Análise de tendência histórica da evolução da hanseníase em Santa Catarina no período de 2001-2015. Arq. Catarin Med, 2018; 47(1): 141-158.

20. PINHEIRO MCG, et al. Conhecimento sobre prevenção de incapacidades em um grupo de autocuidado em hanseníase. REME - Revista Mineira de Enfermagem, 2015; 18(4): 901-906.

21. PREFEITURA MUNICIPAL DE LONDRINA. 2011a. Capacitação identifica danos causados pela hanseníase. Disponível em: https://blog.londrina.pr.gov.br/?p=10481. Acesso em: 20 de out de 2020.

22. PREFEITURA MUNICIPAL DE LONDRINA. 2011b. Reunião e capacitação encerram a Semana da Hanseníase. Disponível em: https://blog.londrina.pr.gov.br/?p=10481. Acesso em: 20 de out de 2020

23. QUARESMA MSM, et al. Perfil clínico e epidemiológico dos pacientes portadores de hanseníase em uma unidade de referência no estado do Paraná. Revista Eletrônica Acervo Saúde, 2019; 18: 1-10.

24. RIBEIRO GC, LANA FCF. Incapacidades físicas em hanseníase: caracterização, fatores relacionados e evolução. Cogitare Enfermagem, 2015; 20(3): 496-503.

25. SANTANA MES. Features of the epidemiological profile of leprosy in a northeast brazilian municipality, from 2014 to 2019. Brazilian Journal of Health Review, 2020; 3(5): 12184-12195.

26. SCHNEIDER PB, FREITAS BHBM. Tendência da hanseníase em menores de 15 anos no Brasil, 2001-2016. Cadernos de Saúde Pública, 2018; 34(3): 1-11.

27. SECRETARIA MUNICIPAL DE SAÚDE DE LONDRINA. 2020. Unidades Básicas de Saúde. Disponível em: https://saude.londrina.pr.gov.br/index.php/unidade-basica-saude.html. Acesso em: 29 out. 2020.

28. SILVA PSR, et al. Perfil clínico-epidemiológico de pacientes portadores de hanseníase em um município do Maranhão. Revista Eletrônica Acervo Saúde, 2020; 12(8): 1-11.

29. SOUZA EA, et al. Hanseníase e gênero no Brasil: tendências em área endêmica da região Nordeste, 2001-2014. Rev. Saúde Pública, 2018; 52(20): 1-12.

30. SOUZA MM, et al. Atributos derivados da atenção primária na assistência ao paciente oncológico. Revista de Enfermagem UFPE on line, 2016; 10(8): 3004-3010.

31. UCHÔA REM, et al. Distribuição dos casos de hanseníase com incapacidade física no estado da Paraíba de 2001 a 2011. Revista de Pesquisa Cuidado é Fundamental Online, 2017; 9(3): 634-640.

32. VIEIRA NF, et al. Qualidade da atenção primária e os efeitos em indicadores de monitoramento da hanseníase. Revista Brasileira de Enfermagem, 2020; 73 (4): 1-8. 